\title{
Article
}

\section{Filler Effects on Temperature Shift Factors in Viscoelastic Properties of Carbon Black Filled Rubbers}

\author{
Yoshinobu ISONO* and Takafumi AOYAMA \\ Department of Materials Science and Technology \\ Nagaoka University of Technology \\ Nagaoka, Niigata 940-2188, Japan \\ (Received : December 06, 2012)
}

\begin{abstract}
Measurements of Relaxation modulus and/or differential dynamic modulus in linear and nonlinear viscoelasticity have been made on cured styrene-butadiene rubber (SBR) 1502 gum and cured SBR containing 20-60 phr carbon black (CB). Linear viscoelastometry has been made at temperatures ranged from -60 to $80{ }^{\circ} \mathrm{C}$ and at frequencies ranged from 0.4 to $100 \mathrm{rad} \mathrm{s}^{-1}$. Nonlinear viscoelastometry has been made with small oscillations at dynamic strain of 0.005 and at frequency of $1.26 \mathrm{rad} \mathrm{s}^{-1}$ superposed on static strains of unity both for shear and extensional deformations. Cured SBR showed $G^{\prime \prime}$ peak temperatures of $-48{ }^{\circ} \mathrm{C}$ at $1.26 \mathrm{rad} \mathrm{s}^{-1}$. The mixing of CB filler showed no effect on the peak temperatures. It was found that all horizontal shift factors can be expressed by single WLF equation in linear viscoelasticity. The horizontal shift factors can be applied for the temperature dependent nonlinear viscoelasticity. Vertical shift factors in linear viscoelasticity depends on filler contents, showing change from entropic elasticity in gum rubber to energetic elasticity of enough filled rubber. Similar change has been found in large shear deformations, but entropic elasticity behaviors in vertical shift factors have been found irrespective of filler contents in large extension. The difference in behaviors of vertical shift factors between large shear and large extension can be understood with the change and recovery of filler network. It was concluded that the horizontal and the vertical shift factors of filled rubbers are governed by matrix polymer and filler network, respectively.
\end{abstract}

Key Words: Filled rubber / Carbon black / Filler network / Time temperature superposition / Dynamic modulus

\section{INTRODUCTION}

Polymeric substances show very wide range of distribution in relaxation time, while experimentally accessible time scale by the use of rheometer is limited to five digits at most. This is a basic reason why time temperature superposition principle (TTSP) is important in polymer ${ }^{1)}$. In an engineering field of filled rubber, however, we have another reason why TTSP is important. For example, loss tangent at $0{ }^{\circ} \mathrm{C}$ and $10 \mathrm{~Hz}$ is widely used as index of wet skid resistance of tire tread rubber $^{2)}$. In the case of wet skid, small deformations at high frequency, typically $10^{4} \mathrm{~Hz}$, at around $30^{\circ} \mathrm{C}$ may be generated between tread and road surfaces. The small deformation of frequency $10^{4} \mathrm{~Hz}$ at $30^{\circ} \mathrm{C}$ may correspond to one at $0{ }^{\circ} \mathrm{C}, 10$ $\mathrm{Hz}$ from the viewpoint of TTSP. ${ }^{1)}$ This is the reason why $\tan \delta$ at $0{ }^{\circ} \mathrm{C}, 10 \mathrm{~Hz}$ (typical) is adopted as a predictor of wet skid resistance. In practical application of TTSP to materials, not only horizontal shift factor, $a_{T}$, but also vertical shift factor, $b_{T}$, must be considered as shown in eqs. 1 and 2 .

\footnotetext{
* Corresponding Author: yisono@nagaokaut.ac.jp
}

$$
\begin{aligned}
& G\left(t, T_{r}\right)=\frac{G\left(t / a_{T}, T\right)}{b_{T}} \\
& G^{*}\left(\omega, T_{r}\right)=\frac{G^{*}\left(\omega a_{T}, T\right)}{b_{T}}
\end{aligned}
$$

where $T_{r}$ is reference temperature, $T$ measured temperature.

Generally speaking, there exist some networks in practical soft materials. Recently existence of filler network in filled rubbers has been recognized experimentally not only in macroscopic scale by rheometry in reversing double-step deformations ${ }^{3)}$ and electric resistance measurement ${ }^{4}$ but also in microscopic scale by in-situ microscopy ${ }^{5,6)}$ and 3D-TEM ${ }^{7-11)}$. Let us think over various networks existing in filled rubbers. Filled rubbers consist of polymer and filler, if we neglect minor additives. So there should exist three networks at least due to three different interactions; polymer-polymer, polymerfiller, and filler-filler interactions as shown schematically in Fig. 1. The first is entanglement and/or crosslinking network due to interchain interaction ${ }^{1)}$. The second is filler network composed of carbon black particles that are joined by bridging polymer chains attached more or less firmly to the particles 
through e.g. entanglement trapped at the filler-polymer interface $^{12-17)}$ (hereafter abbreviated as bridged filler network). The third is filler network by direct particle contact ${ }^{4,12,14)}$ (abbreviated as contact filler network). We may recognize contact filler network where the perimeter distance between the nearest aggregates is $c a .3 \mathrm{~nm}$ and it changes due to large deformation which is observed by 3D-TEM. ${ }^{10,11)}$ Bridged filler network has not been confirmed by experiment. However, it may be plausible, because of the next points. The filler gel ${ }^{12)}$ surrounding carbon black filler particle has been accepted on the basis of the evidence of insoluble fraction of polymers. Its adiabatic compressibility has been estimated to be half of the matrix SBR and four times as high as carbon black. ${ }^{18)}$ Some observations have been reported on nonlinear viscoelasticity due to bonding and debonding between polymer chains and filler surface ${ }^{19)}$ and complex slip between polymers and flat surface due to disentanglement. ${ }^{20)}$ Recently, furthermore, the polymer field theory and the off-lattice computer simulation have predicted the existence and behavior of polymer bridged geletaion. $^{21)}$ Various networks and filler gel may contribute to filler reinforcement.

Temperature dependence of polymer viscoelasticity is independent of molecular weight. This means the temperature dependence of polymer viscoelasticity is determined by that of segmental friction coefficient of matrix polymer. ${ }^{1)}$ The viscoelasticity of polymer systems having entanglement and/ or crosslinking show the same dependence as that of polymer having no long-range intermolecular interaction ${ }^{1,22,23)}$ which indicates entanglement and/or crosslinking have no additional contribution to temperature dependence of segmental friction. Polymer-bridged filler network may be based on constraint on polymer chain by filler gel (in real image, probably by trapped entanglement in filler gel phase $)^{11-16)}$ Therefore, polymerbridged filler and entanglement and/or crosslinking networks are supposed to have no special contribution to temperature dependence of segmental friction.

Friction coefficient $\xi$ of rigid sphere having radius $R$ is determined by matrix viscosity $\eta_{\text {matrix }}$ through Stokes-Einstein equation, $\xi=6 \pi \eta_{\text {matrix }} R$. So the viscosity of the matrix rubber may determine the friction coefficient of filler particle $\xi_{\text {filler }}$. Therefore, temperature dependence of viscoelasticity of filled rubbers, both cured and uncured, may be determined by that of segmental friction coefficient of the matrix polymer and is expected to be independent of kind of fillers and filler content. Therefore, we may expect that horizontal shift factor $a_{T}$ is simply determined by matrix polymer.

In contrast to $a_{T}$, however, temperature dependence of vertical shift factor $b_{T}$ may be complicated. As well known, temperature dependence of $b_{T}$ for polymer changes at glass transition temperature $T_{g}$. Temperature dependent $b_{T}$ curve shows negative slope below $T_{g}$, and positive slope above $T_{g}$. Negative and positive slopes correspond to energetic and entropic eleasticity, respectively. Unfilled polymer shows entropic elasticity above $T_{g}$. At low filler content where bridged filler network is dominant, we have still entropic elasticity. At higher filler content above percolation threshold, however, elastic mechanism should change from entropic to energetic elasticity corresponding to negative slope. In linear viscoelasticity of filled rubber, temperature dependent $b_{T}$ curve may shows initial positive slope behavior followed by negative slope behavior as the $\mathrm{CB}$ content increases.

In nonlinear viscoelasticity of $\mathrm{CB}$ filled rubber, additional difference in $b_{T}$ may be observed between large shear and large elongation. Both large shear and large extension leads to rupture in contact filler network. In large shear, however, reformation of contact filler network may be expected ${ }^{4)}$. Hence temperature dependent $b_{T}$ curve for rubber having high $\mathrm{CB}$ content may show negative slope in large shear, but may not show in large extension.

The purpose of this work is to test the idea on horizontal and vertical shift factors mentioned above by measuring the temperature-dependent linear and nonlinear viscoelasticity of various carbon black filled, cured rubbers.

Table 1. Sample formulations (unit in phr)

\begin{tabular}{|c|c|c|c|c|c|}
\hline Sample No. & 1 & 2 & 3 & 4 & 5 \\
\hline SBR1502 & 100 & 100 & 100 & 100 & 100 \\
\hline CB (N330) & & 20 & 35 & 40 & 60 \\
\hline $\mathrm{ZnO}$ & 3 & 3 & 3 & 3 & 3 \\
\hline Steraric Acid & 2 & 2 & 2 & 2 & 2 \\
\hline Anti-Oxidant ${ }^{a}$ & 1 & 1 & 1 & 1 & 1 \\
\hline Sulfur & 1.5 & 1.5 & 1.5 & 1.5 & 1.5 \\
\hline Accelerator $\mathrm{CBS}^{\mathrm{b})}$ & 1 & 1 & 1 & 1 & 1 \\
\hline
\end{tabular}

\footnotetext{
a) $\mathrm{N}$-(1,3-dimethylbutyl)-N'-phenyl- $p$-phenylenediamine

b) N-Cyclohexyl-2-benzothiazolylsulfenamide
} 


\section{EXPERIMENTAL}

\subsection{Materials}

The rubber used is styrene-butadiene random copolymer (Nipol SBR1502, $\mathrm{M}_{\mathrm{w}}=4.3 \times 10^{5}, T_{g}=-52^{\circ} \mathrm{C}$, Zeon Corporation). Carbon black (CB) used was N330 (SEAST 3, Tokai Carbon) having average primary particle sizes of $28 \mathrm{~nm}$. Other additives are zinc oxide (Shodo Chemical), Antioxidant, $\mathrm{N}$-(1,3-dimethylbutyl)-N'-phenyl- $p$-phenylenediamine (6PPD, Ouchi Shinko Chemical Industrial), stearic acid (Stearic Acid N for industrial use, Asahi-Denka), sulfur (powdered, 200 mesh, Tsurumi Chemical Industry) and accelerator, N-cyclohexyl-2-benzothiazolylsulfenamido (CBS, Ouchi Shinko Chemical Industrial). Details on compound formulation are shown in Table I, where unit "phr" means $\mathrm{g}$ of additives per $100 \mathrm{~g}$ of raw rubber. CB master batches are prepared in 1L Kneader (DS1-5MHB-S, Moriyama) for 3.5 minutes at $343 \mathrm{~K}$ in the beginning (at ca. $390 \mathrm{~K}$ in the final stage, depending on compounds). The curatives are mixed to the master batches using 6 inches two-roll mill (Kansai Roll). The compounds are cured for 30 minutes at $433 \mathrm{~K}$ under a pressure of $3 \mathrm{MPa}$.

\subsection{Measurement}

\subsubsection{Volume resistivity}

Electrical resistance was measured with digital electrometer R8340A (ADC). In order to take electrical contact, the sample was fixed to the disc-shaped electrode of $10 \mathrm{~mm}$ in diameter made of stainless steel plates in a Teflon mold by fusion at $353 \mathrm{~K}$ for 2 hours followed by vulcanization at $433 \mathrm{~K}$ for $30 \mathrm{~min}$. Resistance measured was converted to volume resistivity $\boldsymbol{R}$ with eq. (3)

$$
\boldsymbol{R}=R(A / h)
$$

Where $R$ is resistance measured, $A$ sample contact area, and $h$ sample height.

\subsubsection{Dynamic modulus in linear viscoelasticity}

Dynamic modulus in linear viscoelasticity was measured with high-precision Rotational Rheometer ARES-G2 (TA Instruments). A rectangular-shaped specimen ( $8 \mathrm{~mm}$ in width, $30 \mathrm{~mm}$ in length, and $2 \mathrm{~mm}$ in thickness) was deformed in torsion. The frequency of the oscillations ranged from 0.4 to $100 \mathrm{rad} \mathrm{s}^{-1}$. The temperature ranged from -56 to $80{ }^{\circ} \mathrm{C}$ for frequency-temperature dispersion measurements and from -60 to $80{ }^{\circ} \mathrm{C}$ for temperature dispersion measurements at $1.26 \mathrm{rad} \mathrm{s}^{-1}$. The temperatures changed by $2-5^{\circ} \mathrm{C}$ step with accuracy of $\pm 0.1^{\circ} \mathrm{C}$. The dynamic shear strain amplitude was $5.0 \times 10^{-3}$ at maximum.

\subsubsection{Dynamic modulus in nonlinear viscoelasticity}

The apparatus used for nonlinear viscoelastometry was biaxial rheometer made by ourselves. ${ }^{24-26)}$ It is equipped with servo-controlled oil-hydraulic actuator (JT Toshi), a load cell (LRM-50K, Nihon Tokushu Sokki), and a cantilever spring type displacement transducer (CE-10, Tokyo Sokki). In addition, the apparatus is equipped with special air bearings to prevent undesired rotation of hydraulic actuator around the axis.

The measurements of differential dynamic modulus were made in two different deformation modes. The one was made by small oscillations along axis with strain amplitude of $1 \times 10^{-3}-2 \times 10^{-3}$ at frequency $1.26 \mathrm{rad} \mathrm{s}^{-1}$ superposed on a step shear strain 1.0 at 30 to $80{ }^{\circ} \mathrm{C}$ by the use of discshaped sample specimen of $10 \mathrm{~mm}$ in diameter and $2 \mathrm{~mm}$ in thickness. The other was by small oscillations along axis with strain amplitude of $3 \times 10^{-3}$ to $5 \times 10^{-3}$ at frequency $1.26 \mathrm{rad}$ $\mathrm{s}^{-1}$ superposed on a step extensional strain 1.0 at 30 to $80{ }^{\circ} \mathrm{C}$ by the use of rectangular-shaped specimen $(8 \mathrm{~mm}$ in width, $30 \mathrm{~mm}$ in length, and $2 \mathrm{~mm}$ in thickness).

\section{RESULTS AND DISCUSSION}

Figure 2 shows CB filler concentration dependence of volume resistivity $\rho$ of unfilled, cured and filled, cured SBRs at no deformation. The value of $\rho$ for unfilled, cured SBR was very high value of $8.4 \times 10^{10} \Omega \mathrm{m}$. At low concentration of $20 \mathrm{phr}$ filled, cured SBR showed $1.3 \times 10^{11} \Omega \mathrm{m}$ similar to the value for unfilled, cured SBR, indicating no formation of contact filler network. With the change in CB loading from 20 to $35 \mathrm{phr}$, however, resistivity showed decrease by around six orders of magnitude, followed by gradual decrease with increase in $\mathrm{CB}$ concentration. The data indicate that percolation threshold exists between 20 and $35 \mathrm{phr}$.

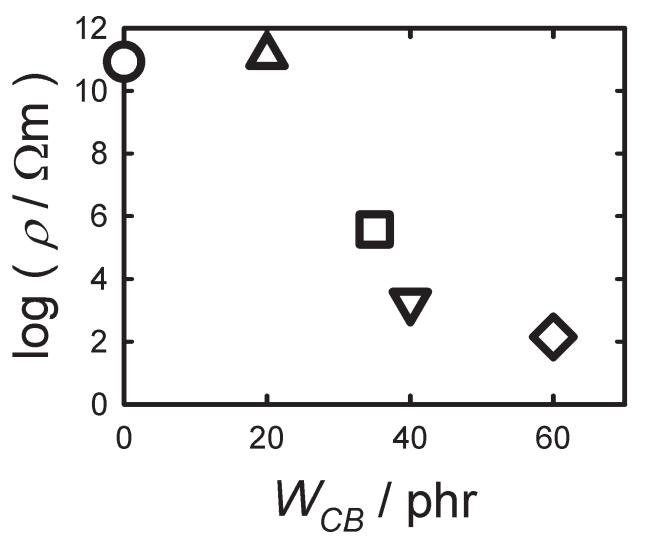

Fig. 2. Volume resistivity of carbon black filled, cured SBRs. 
As being found by Kojiya and $\mathrm{Katoh}^{10)}$, the perimeter distance between the nearest aggregates for the $\mathrm{CB}$ filled rubber percolated was ca. $3 \mathrm{~nm}$ irrespective of $\mathrm{CB}$ concentration. So decrease in resistivity at high $\mathrm{CB}$ concentration may imply increase in number of contact filler network strands. The CB concentration dependence of resistivity shown in Fig. 2 may support such speculation.

Percolation threshold volume fraction $\varphi_{C}$ may depend on filler particle form and spatial distribution. ${ }^{13)}$ Under an assumption of simple cubic lattice of spherical filler, $\varphi_{C}$ is given by ${ }^{27)}$

$$
\varphi_{C}=1 /(1+4 d v)
$$

where $d$ is filler density and $v$ specific void space of random dense packed filler. Use of the values $d=1.8 \mathrm{~g} \mathrm{~cm}^{-3}$ and $v=0.98 \mathrm{~cm}^{3} \mathrm{~g}^{-1}$ for $\mathrm{N} 330$ carbon black ${ }^{27)}$ gives $\varphi_{C}=0.124$ (28 phr), which is not only close to the values reported ${ }^{4)}$ but also in agreement with the value observed in the present study.

The temperature-dependent curves of dynamic modulus and loss tangent at $1.26 \mathrm{rad} \mathrm{s}^{-1}$ for all samples are shown in Fig. 3. The curves are shifted along the ordinate by a factor of A denoted in the panels in order to avoid overlap of curves. In Fig. 3, clear peaks in loss modulus $G^{\prime \prime}$ and loss $\operatorname{tangent} \tan \delta$ can be observed. It can be found that $G^{\prime \prime}$ shows the peaks at $-48{ }^{\circ} \mathrm{C}$ for unfilled, cured and filled, cured samples. The $G^{\prime \prime}$ peak temperatures for filled, cured samples are independent of filler content as expected. The peaks in $\tan \delta$ are found at $-41{ }^{\circ} \mathrm{C}$ for all samples, which differ from those observed for $G^{\prime \prime}$.

Loss tangent $\tan \delta$ is defined by ratio of loss modulus $G^{\prime \prime}$ to storage modulus $G^{\prime}$. It is a measure of energy loss on the basis of energy storage. $G^{\prime}$ shows decrease by around 2.5 orders of magnitude at glass-to-rubber transition region. So $\tan \delta$ peak temperature appears at the higher temperature side. Loss energy in unit period of cyclic deformations can be given by $\pi \sigma_{0} \gamma_{0} \sin \delta$, where $\sigma_{0}, \gamma_{0}$, and $\delta$ means peak stress, peak strain and phase angle difference between stress and strain waves, which can be approximated by $\pi \sigma_{0} \gamma_{0} \tan \delta$ only in the elasticity-rich limit. Hence the peak temperature in $G^{\prime \prime}$ should be more important physically than that in $\tan \delta$.

As mentioned above, $\tan \delta$ is dimensionless quantity. So it is independent of the temperature shift in magnitude. Therefore we can determine the temperature shift in frequency $\left(a_{T}\right.$, the usual ratio of relaxation times) by using $\tan \delta$ curves. Storage and loss modulus master curves can be obtained by temperature shifts in frequency of the amounts of $a_{T}$, followed by the shifts in magnitude. Figure 4 shows the dynamic modulus and loss tangent master curves obtained through the method explained above for the samples 1-5 in linear viscoelasticity. Here the reference temperatures were taken at the $G^{\prime \prime}$ peaks. Good superposition was obtained for $G^{\prime}, G^{\prime \prime}$, and $\tan \delta$ curves. By comparing the master curves in Fig. 4 , it is found that storage and loss modulus increases with addition of carbon black filler, and peaks in $G^{\prime \prime}$ and $\tan \delta$ curves are located at the same frequencies, 0 and -1.3 in $\log \omega$, respectively. This means that the mixing of fillers has no effect on the locations in frequency of $G^{\prime \prime}$ and $\tan \delta$ peaks, although filler mixing has effects on the magnitude of dynamic modulus.

From the above results, it is clear that the TTSP can be applied to all samples. Furthermore, it has been reported that the peak temperatures or the peak frequencies of $G^{\prime \prime}$ and $\tan \delta$ are dependent of intermolecular cross-linking, but independent
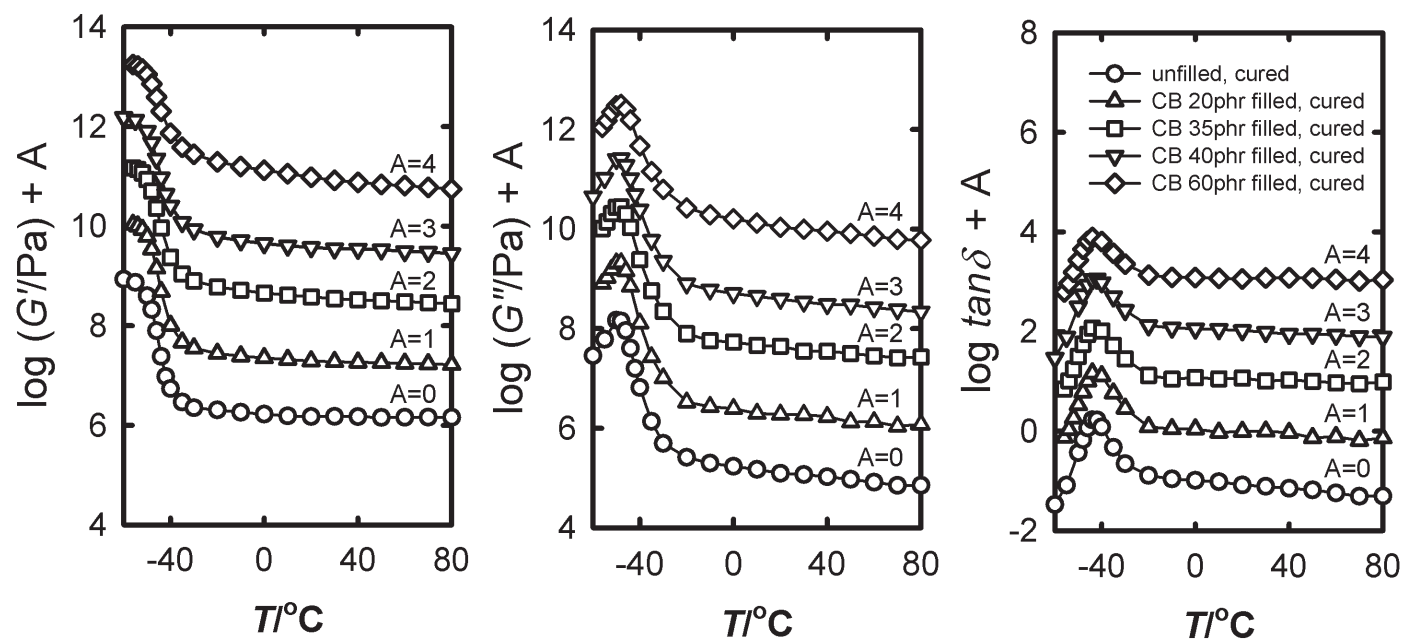

Fig. 3. Dynamic modulus of various filled, cured SBRs at $1.26 \mathrm{rad} \mathrm{s}^{-1}$ plotted against temperature. The curves are shifted along ordinates by a factor of A denoted in the panels. 
of kind of fillers. ${ }^{28)}$ Taking the facts into consideration, it can be expected that the temperature dependence of viscoelastic properties of the samples 1-5 is expressed by universal function being independent of carbon black filler content, if we take appropriate reference temperatures $T_{r}$. Figure 5 shows the semi-logarithmic plot of $a_{T}$ for the samples 1-5 against the temperatures difference, $T-T_{r}$, where $T_{r}$ is taken at the $G^{\prime \prime}$ peak temperatures in Fig. 3. The shift factors $a_{T}$ can be reduced as expected.

The solid line in Fig. 5 denotes the WLF curve. All shift factors $a_{T}$ can be well expressed by the WLF equation. It may be concluded that the matrix polymer determines the temperature dependence in the linear viscoelasticity of the present rubber samples as preliminarily discussed in the introduction.

On the other hand, temperature dependence in vertical shift factor $b_{T}$ is much different from that in $a_{T}$ as shown in Fig. 6 . $b_{T}$ curves are independent of filler content below $T_{r}$ where $b_{T}$ is determined by matrix polymer. However $b_{T}$ depends on filler content above $T_{r}$. That is, $b_{T}$ curves show positive slope
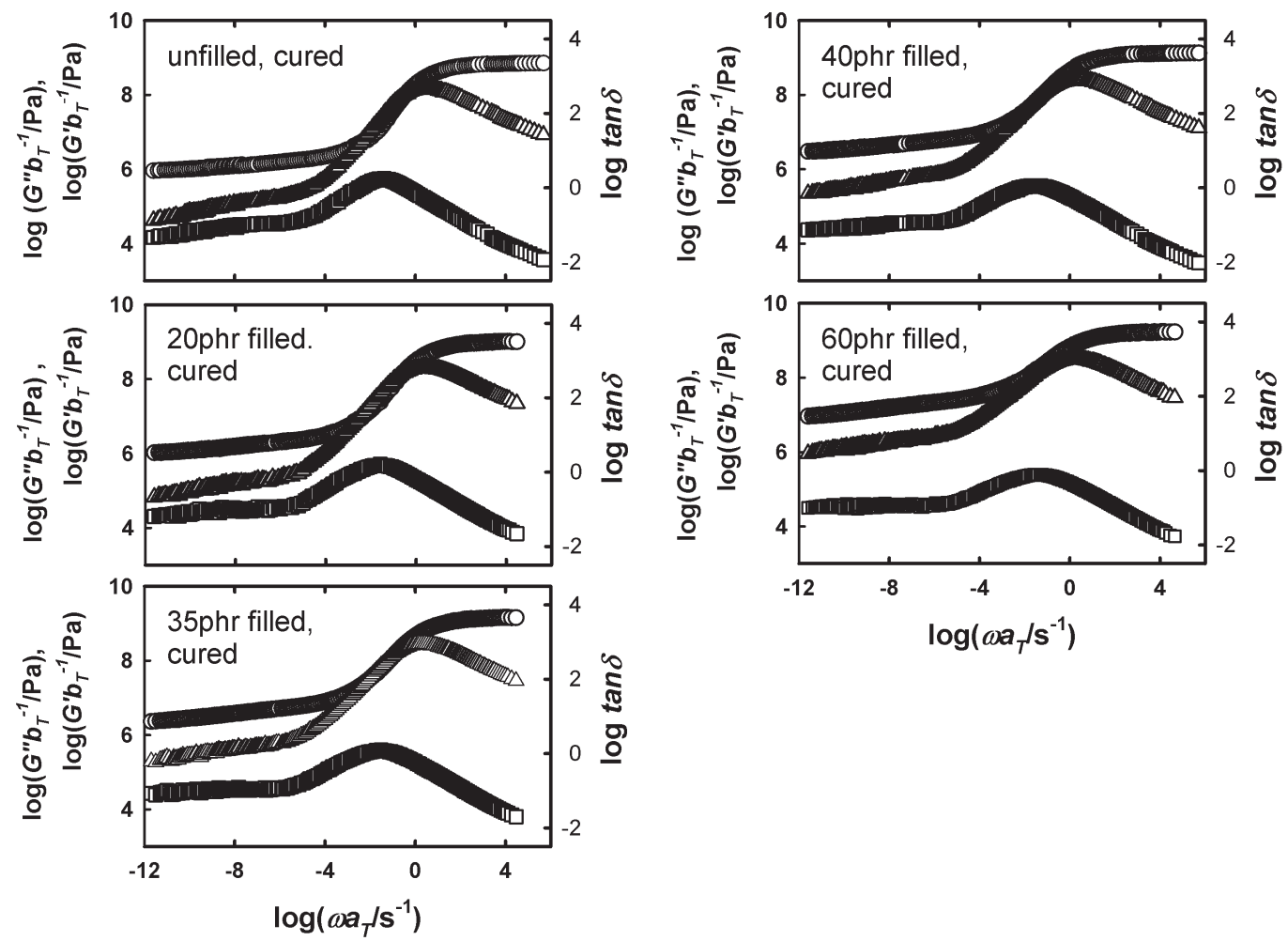

Fig. 4. Storage modulus $(\bigcirc)$, loss modulus $(\triangle)$, and loss tangent $(\square)$ master curves in linear viscoelasticity for various filled, cured SBRs at $-48^{\circ} \mathrm{C}$.

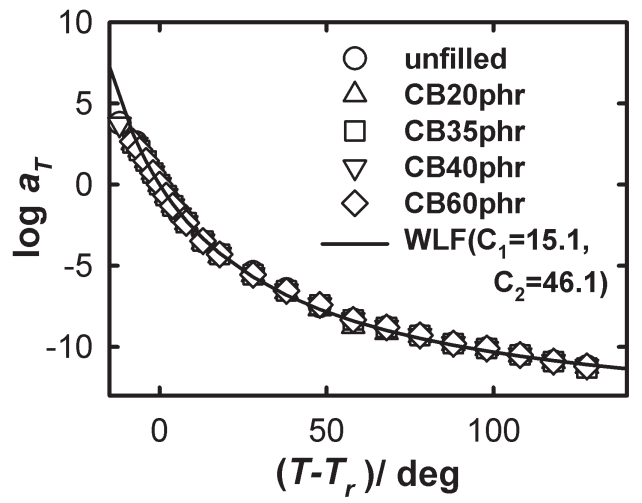

Fig. 5. Horizontal shift factors $a_{T}$ plotted semi-logarithmically against $T-T_{r}$ for unfilled, cured and various filled, cured SBRs. The value of $T_{r}$ was chosen at $G^{\prime \prime}$ peak temperature, $-48^{\circ} \mathrm{C}$.

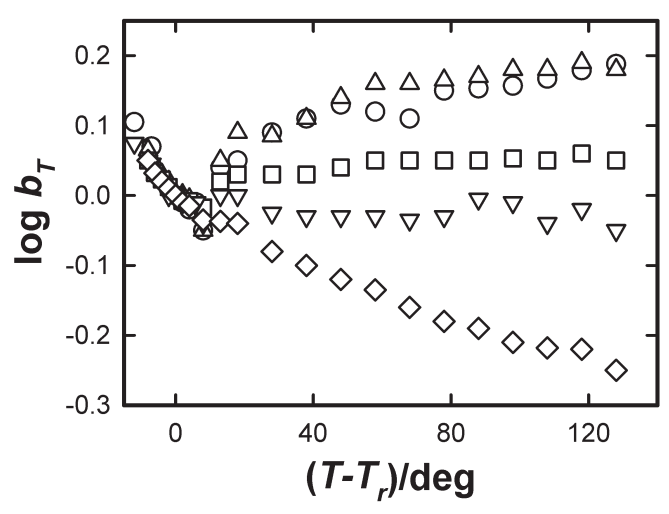

Fig. 6. Vertical shift factors $b_{T}$ plotted semi-logarithmically against $T-T_{r}$ for unfilled, cured $(\bigcirc), 20 \mathrm{phr}(\triangle), 35 \mathrm{phr}(\square), 40 \mathrm{phr}(\nabla)$, and $60 \mathrm{phr}(\diamond)$ filled, cured SBRs. The value of $T_{r}$ was chosen at $G^{\prime \prime}$ peak temperature, $-48^{\circ} \mathrm{C}$. 
corresponding to entropic elasticity at lower CB content, but do negative slope corresponding to energetic elasticity at higher $\mathrm{CB}$ content. Curves of $b_{T}$ shows weak temperature dependence near percolation threshold in $\rho-W_{C B}$ relationship where contact filler network begins to be formed. The change from positive to negative slopes in $b_{T}-T$ curves observed in Fig. 6 support the idea on hierarchical structures in filled (a) in shear at $\gamma=1$

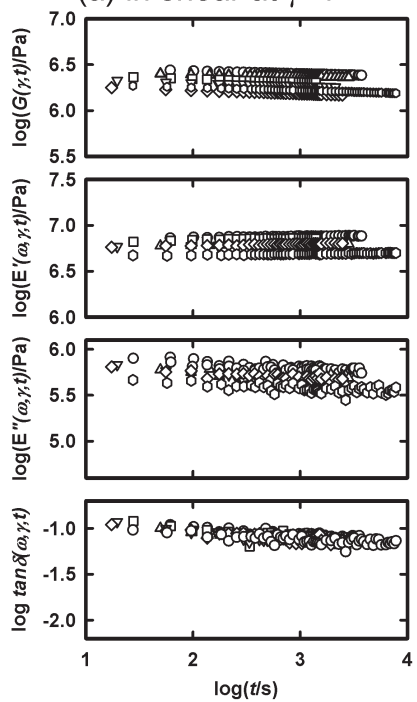

(b) in elongation at $\varepsilon=1$

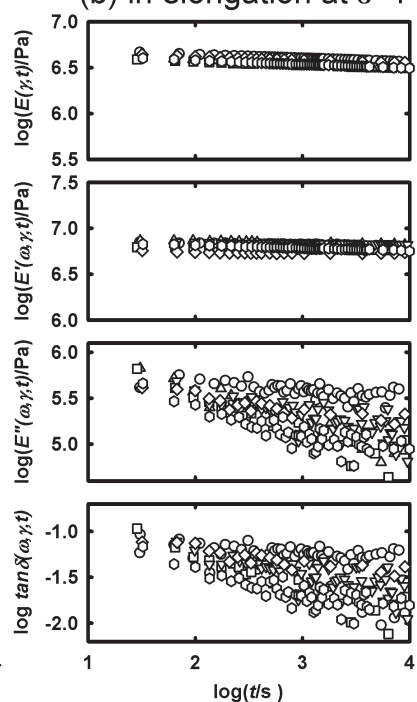

Fig. 7. Relaxation modulus and differential dynamic modulus of $\mathrm{CB}$ 40phr filled, cured SBR (a) in shear (a) at $\gamma=1$ and (b) in elongation at (b) $\varepsilon=1$ at various temperature $\left(\bigcirc 30^{\circ} \mathrm{C}, \triangle 40^{\circ} \mathrm{C}\right.$, $\square 50^{\circ} \mathrm{C}, \nabla 60^{\circ} \mathrm{C}, \diamond 70^{\circ} \mathrm{C}$, hexagon $\left.80^{\circ} \mathrm{C}\right)$. rubber preliminary discussed in the introduction.

Figures 7(a) and 7(b) show relaxation modulus and differential dynamic modulus of carbon black 40 phr filled, cured SBRs in large shear at $\gamma=1$ and those in large extension at $\varepsilon=1$ at various temperatures. As preliminarily discussed in the introduction, horizontal shift factor $a_{T}$ in linear viscoelasticity is determined by matrix polymer through friction coefficient not only for unfilled, cured but also for filled, cued rubbers. The situation should be unaltered even in nonlinear viscoelasticity. At first, hence, the individual curve at different temperature is shifted horizontally by the value of $a_{T}$ shown in Fig. 5, then shifted vertically till $E^{\prime}$ curves are superposed. The master curves obtained in this way are shown in Figs. 8 and 9. Good superposition was obtained for $G(t)$ and $E^{\prime}$ curves in large shear and also for $E(t)$ and $E^{\prime}$ curves in large extension, although $E^{\prime \prime}$ and $\tan \delta$ curves are scattered especially in large extension. Vertical shift factors in nonlinear viscoelasticity are shown in Fig. 10. Data of $b_{T}$ show weak temperature dependence at lower $\mathrm{CB}$ content both in shear and in extension. However, clear difference has been found for higher $\mathrm{CB}$ content as expected. All samples under large extension exhibit weak temperature dependence even at higher $\mathrm{CB}$ content such as $60 \mathrm{phr}$, but the sample under large shear at $40 \mathrm{phr}$ shows negative slope in $b_{T}-T$ curve corresponding to energetic elasticity. This is because contact filler network is ruptured in large extension, while it is reformed in large shear. (a) unfilled, cured

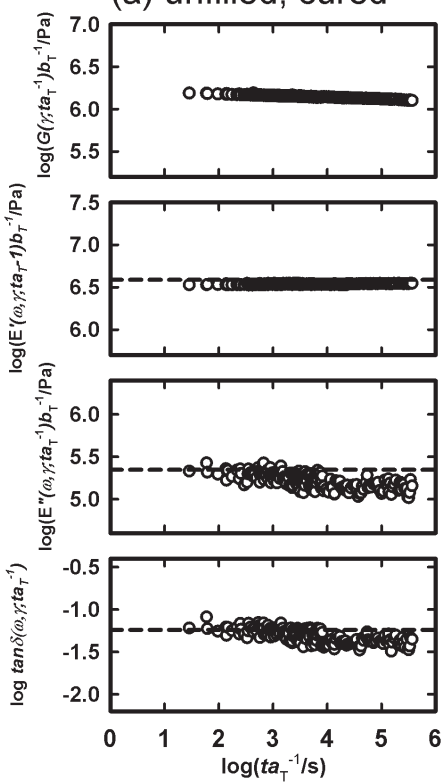

(b) 20phr filled, cured

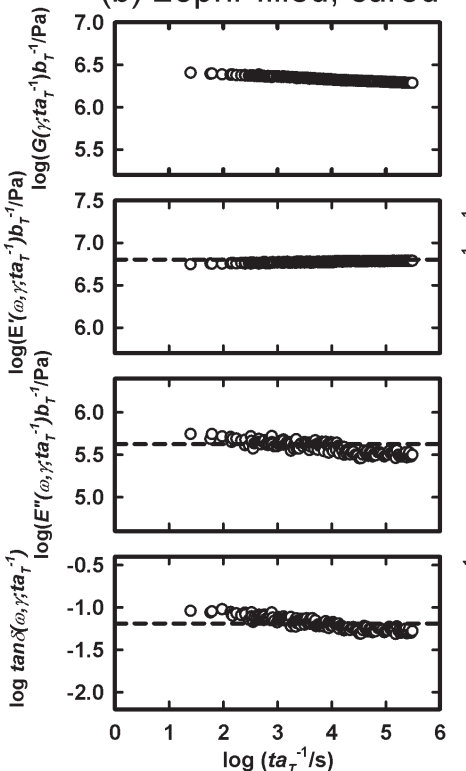

(c) 40 phr filled, cured

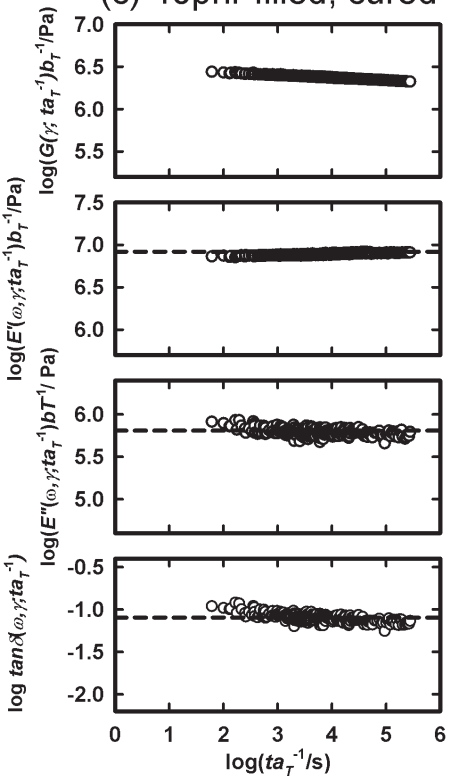

Fig. 8. Shear relaxation modulus and differential dynamic modulus master curves at $30^{\circ} \mathrm{C}$ for (a) unfilled, cured, (b) $20 \mathrm{phr}$ filled, cured, and (c) $40 \mathrm{phr}$ filled, cured SBRs. 


\section{CONCLUSION}

Temperature dependence of Relaxation modulus and/or differential dynamic modulus have been studied on cured SBR gum and cured SBR containing 20-60 phr carbon black (CB). Cured SBR gum showed $G^{\prime \prime}$ peak temperatures of $-48{ }^{\circ} \mathrm{C}$ at $1.26 \mathrm{rad} \mathrm{s}^{-1}$. The mixing of CB filler showed no effect on the $G^{\prime \prime}$ peak temperatures. It was found that all horizontal shift factors can be expressed by single WLF equation in linear viscoelasticity. The horizontal shift factors can be applied for the temperature dependent nonlinear viscoelasticity. Vertical shift factors in linear viscoelasticity depends on filler contents, showing change from entropic elasticity in gum rubber to energetic elasticity of enough filled rubber. Similar change has been found in large shear deformations, but entropic elasticity behaviors in vertical shift factors have been found irrespective of filler contents in large extension. The difference in behaviors of vertical shift factors between large shear and large extension can be understood with the change and recovery of filler network. It was concluded that the horizontal and the vertical shift factors of filled rubbers are governed by matrix polymer and filler network, respectively.

\section{Acknowledgements}

This work was supported in part by Grant-in-Aid for Scientific Research (B) (No.21360330) from the Ministry of Education, Science, Sports and Culture, Japan. (a) 20phr filled, cured
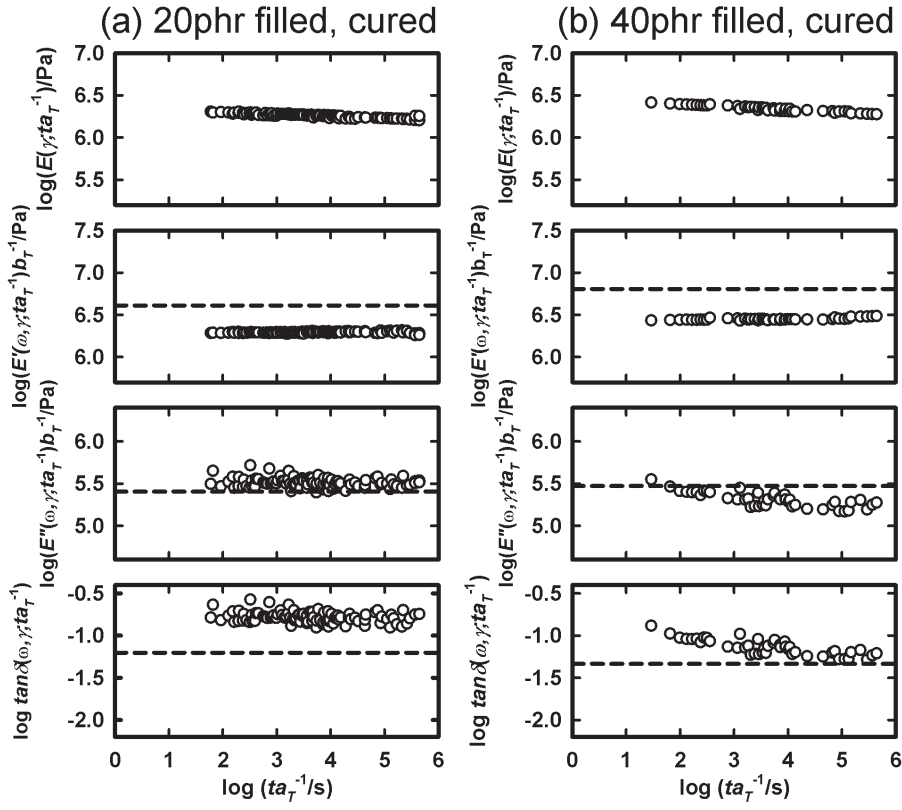

(c) 60 phr filled, cured

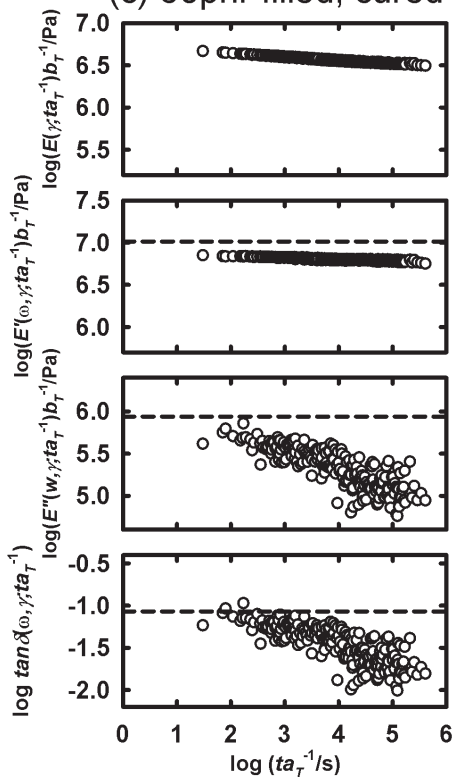

Fig. 9. Extensional relaxation modulus and differential dynamic modulus master curves at $30{ }^{\circ} \mathrm{C}$ for (a) $20 \mathrm{phr}$ filled, cured, (b) $40 \mathrm{phr}$ filled, cured, and (c) $60 \mathrm{phr}$ filled, cured SBRs.

(a) in shear at $\gamma=1$

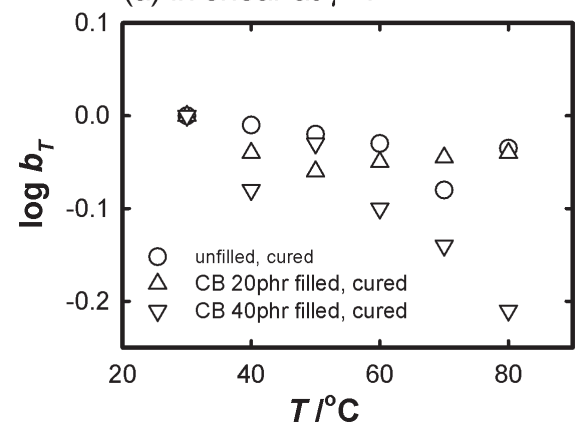

(b) in elongation at $\varepsilon=1$

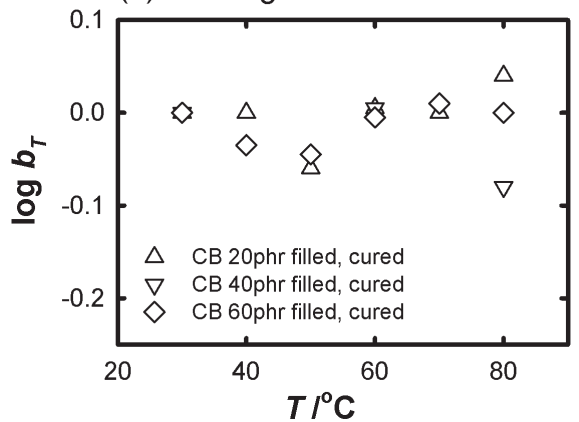

Fig. 10. Temperature dependence of vertical shift factors $b_{T}$ in shear at $\gamma=1$ (left) and those in elongation at $\varepsilon=1$ (right). 


\section{REFERENCES}

1) Ferry J D, "Viscoelastic property of polymers", 3rd ed, (1980), Willy \& Sons, Inc, N.Y.

2) Akutagawa K, Ozawa Y, Yamada Y, Hamada T, Nippon Gomu Kyokaishi, 80, 394 (2007).

3) Satoh $\mathrm{Y}$, Suda K, Fujii S, Kawahara S, Isono Y, Kagami S, e-J. Soft Mater, 3, 14 (2007).

4) Satoh $Y$, Fujii $S$, Kawahara S, Isono Y, Kagami S, e-J. Soft Mater, 3, 29 (2007).

5) Suh YJ, Friedlander SK, J Appl Phys, 93, 3515 (2003).

6) Rong W, Pelling AE, Ryan A, Gimzewski JK, Friedlander SK, Nano Letters, 4, 2287 (2004).

7) Ikeda Y, Katoh A, Shimanuki J, Kohjiya S, Macromolecular Rapid Communications, 25, 1186 (2004).

8) Kohjiya S, Katoh A, Shimanuki J, Hasegawa T, Ikeda Y, J Mater Sci, 40, 2553 (2005).

9) Kohjiya S, Katoh A, Shimanuki J, Hasegawa T, Ikeda Y, Polymer, 46, 4440 (2005).

10) Kohjiya S, Katoh A, Suda T, Shimanuki J, Ikeda Y, Polymer, 47, 3298 (2006).

11) Katoh A, Isono Y, J Appl Polym Sci, 128, 2498 (2013).

12) Medalia A, Rubber Chem Technol, 51, 437 (1978).

13) Klueppel M, Adv. Polym Sci, 164, 1-86 (2003).
14) Isono Y, Ferry JD, Rubber Chem Technol, 57, 925 (1984).

15) Sternstein SS, Zhu AJ, Macromolecules, 35, 7262 (2002).

16) Froehlich J, Niedermeier W, Luginsland HD, Composites, Part A: Applied Science and Manufacturing, 36, 449 (2005).

17) Raos G, Moreno M, Elli S, Macromolecules, 39, 6744 (2006).

18) Maebayashi M, Endo M, Matsuoka $T$, Koda $S$, Isono $Y, e-J$. Soft Mater, 1, 26 (2005).

19) Wang SQ, Inn YW, Rheologica Acta, 33, 108 (1994).

20) Leger L, Raphael E, Hervet H, Adv Polym Sci, 138, 185 (1999).

21) Surve M, Pryamitsyn V, Ganesan V, Phys Rev Lett, 96, 177805 (2006).

22) Isono $Y$, Fujimoto T, Takeno N, Kajiura, H, Nagasawa M, Macromolecules, 11, 888 (1978).

23) Isono $\mathrm{Y}$, Itoh K, Komiyatani T, Fujimoto T, Macromolecules, 24, 4429 (1991).

24) Isono Y, Nishitake T, Polymer, 36, 1635 (1995).

25) Isono $\mathrm{Y}$, Kamohara T, Takano A, Kase T, Rheol Acta, 36, 245 (1997).

26) Isono Y, Kawahara S, Kase T, Nihon Rheoroji Gakkaishi, 31, 201 (2003).

27) Janzen J, J Appl Phys, 46, 966 (1975).

28) Wu J, Fujii S, Kawahara S, Isono Y, e-J. Soft Mater, 4, 41 (2008). 